\title{
Extreme wave loads on monopile substructures: precomputed kinematics coupled with the pressure impulse slamming load model
}

Pierella, Fabio; Ghadirian, Amin; Bredmose, Henrik

Published in:

Proceedings of International Offshore Wind Technical Conference 2019

Link to article, DOI:

10.1115/IOWTC2019-7618

Publication date:

2019

Document Version

Peer reviewed version

Link back to DTU Orbit

Citation (APA):

Pierella, F., Ghadirian, A., \& Bredmose, H. (2019). Extreme wave loads on monopile substructures: precomputed kinematics coupled with the pressure impulse slamming load model. In Proceedings of International Offshore Wind Technical Conference 2019 American Society of Mechanical Engineers. https://doi.org/10.1115/IOWTC2019-7618

\section{General rights}

Copyright and moral rights for the publications made accessible in the public portal are retained by the authors and/or other copyright owners and it is a condition of accessing publications that users recognise and abide by the legal requirements associated with these rights.

- Users may download and print one copy of any publication from the public portal for the purpose of private study or research.

- You may not further distribute the material or use it for any profit-making activity or commercial gain

- You may freely distribute the URL identifying the publication in the public portal 


\title{
Extreme Wave Loads On Monopile Substructures: Precomputed Kinematics Coupled With The Pressure Impulse Slamming Load Model
}

\author{
Fabio Pierella ${ }^{1}$, Amin Ghadirian ${ }^{1}$, and Henrik Bredmose ${ }^{1}$ \\ ${ }^{1}$ DTU Wind Energy, , Technical University of Denmark, Nils \\ Koppels Allé, Building 403,, DK-2800 Kgs. Lyngby , Email: \\ fabpi@dtu.dk
}

IOWTC 2019, 3-6 November 2019, Malta

\begin{abstract}
Monopiles are nowadays the preferred substructure type for bottomfixed offshore wind turbines at shallow to intermediate water depths. At these locations, the large waves that contribute to extreme loads are strongly nonlinear. Therefore they are not easily reproduced via the simple engineering models who are commonly used in the offshore industry. In the current approach, we develop a design pattern which improves this standard methodology.

To retain nonlinearity in the force computations, we have precomputed a number of wave realizations by means of a potential fully-nonlinear code (OceanWave3D), for a wide span of nondimensional water depths and significant wave heights. The designer can then extract a wave kinematics time series from the precomputed set, scale it by the Froude law, and couple it with a suitable force model to compute loads. To complete the picture, slamming loads are calculated by means of the so-called pressure impulse model, recently developed at DTU. Rather than computing the time series of the slamming load, the model uses a few parameters, all except one determinable from the incident wave to calculate the pressure impulse.

First comparisons with experimental results, obtained in the framework of the DeRisk project, are promising. The force and the wave elevation statistics from the precomputed simulations are in good agreement with the experiments. Some discrepancies are present, due to an imperfect scaling and to the differences in the physical and numerical domains. The computed loads from the slamming model match the experimental ones quite closely, when the wave celerity is extracted as the ratio between the time gradient and the $x$-wise space gradient of the surface elevation.
\end{abstract}




\section{INTRODUCTION}

When designing offshore wind turbines installed on monopiles support structures, it is vital to correctly estimate ultimate load states (ULS) coming from extreme waves to avoid overly conservative safety factors or nonconservative designs.

At the depths at which monopiles are typically installed $(20[m] \div 40[m])$, large waves that occur during severe sea states have typical nonlinear characteristics: they are three-dimensional, their crests are not symmetric, and they are prone to breaking. Especially in parked conditions, when the aerodynamic damping is particularly low, loads from these wave events can become design drivers. The kinematics of these waves is not easily described, unless a complex model is used. For example, Oggiano et al. [1] reproduced nearly-breaking long-crested waves from the WaveLoads experimental campaign [2] via Computational Fluid Dynamics (CFD), achieving a close agreement both for waves and the force induced on a monopile. However, complex models may be too computationally heavy for the designer that wishes to achieve a quick result for a large number of test cases.

Current norms for design of the offshore wind turbine substructures, such as IEC-61400-3 [3] suggest some engineering approaches to calculate ULS that allow to retain both the nonlinearity and the stochasticity of the process in a simplified way. In the constrained wave approach, a 50-year regular extreme wave is embedded into an irregular background linear sea realization. The extreme wave is usually a regular stream function wave [4. A number of methodologies to smoothly embed the stream function inside the background linear field are available in the literature [5, 6. However, stream function waves are derived on the assumption of flat bed, are symmetric about their crests, and have a deterministic behavior. Moreover, the largest force or moment response on a flexible structure is not always associated with the highest wave, as it also depends on the dynamic response of the structure.

In addition to the above mentioned loads, waves breaking in the proximity of a structure also exert an impulsive load, called slamming force. The load is generated by a sudden exchange of momentum between the slamming front of the wave and the structural wall. The impact load can be seen as an additional, fast-paced load cycle that happens on top of the quasi-static force cycle induced by the non-breaking underlying wave on the structure. Slamming loads on cylinders can be predicted by a number of engineering models [7, 8]. However, comparisons with experimental results [9] show that these models are quite sensitive on the modelling assumptions.

More recently, the researchers involved in the WiFi Joint Industry Project [10] have numerically and experimentally investigated breaking wave loads on monopiles and proposed a validated model. The model takes into account the impact force by increasing the height of a stream function wave by a suitable factor. Despite this approach is fast and easy to apply, it does not seem to be linked with the underlying physics regulating the momentum exchange between water and structure. 
The objective of the current work is therefore to improve the accuracy in the computation of extreme loads in shallow to intermediate waters by introducing a new procedure. In the first part of the new strategy, we generate a precomputed set of wave realizations by means of a potential fully-nonlinear code (OceanWave3D [11]), covering a wide span of nondimensional water depths $(h)$ and significant wave heights $\left(H_{S}\right)$. Rather than performing own computations, the designer extracts wave kinematics time series from the precomputed set and calculates the forcing on a structure via a suitable load model (for example the Morison [12, the Rainey [13] or the more recent Fluid-Impulse [14] models).

Secondly, to further increase the level of physics detail, the designer calculates slamming loads from wave breaking, using the new approach developed at DTU by Ghadirian and Bredmose [15. From this model, rather than the force time series associated with a certain impact, the user computes the time integrated pressure impulse, and consequently the surface integrated force impulse. Preliminary results show that the model overpredicts the pressure impulse from a slamming impact on a cylinder computed via CFD by only $3 \%$. More validation was also conducted against experiments [16] and consistent results were obtained.

The objective of this paper is to exemplify a typical procedure for computing extreme wave loads on a cylinder via our methodology. The results will be compared to wave tank experimental results obtained in the framework of the DeRisk project [17.

\section{METHODOLOGY}

\section{THE COMPUTED KINEMATICS}

The fundamental idea behind the current methodology is to pre-compute a set of extreme sea state realizations via the fully-nonlinear potential code OceanWave3D [11. The runs were all generated on a bidimensional, shoaling computational domain $L=25 \cdot 10^{3}[\mathrm{~m}]$ long. The domain, in Figure 1. was $h_{\text {in }}=250[\mathrm{~m}]$ deep at the inlet (offshore) and $h_{\text {out }}=12.5[\mathrm{~m}]$ at the outlet (onshore). While the first and last $2.5 \cdot 10^{3}[\mathrm{~m}]$ were characterized by a flat bottom, the central $20 \cdot 10^{3}[\mathrm{~m}]$ have a gentle 1:100 tangent hyperbolic slope. The shape of the sea bottom was calculated via the following equation:

$$
h(x)= \begin{cases}250 & x^{*}=\frac{x-2.5 \cdot 10^{3}[m]}{20 \cdot 10^{3}[m]} \\ 250\left(1-\frac{\tanh \left(\sin \left(\pi x^{*}\right)\right.}{2\left(1-4 x^{* 2}\right)}\right), & 0<x^{*} \leq 1 \\ 12.5 & x^{*}>1\end{cases}
$$

To generate the $2 \mathrm{D}$ wave realizations, object of the current study, a number of linear sea states were imposed in a relaxation zone adjacent to the inlet 
boundary. As the waves travel towards the shore, they start to interact and become increasingly nonlinear. An initial warm-up time of 2 hours was allowed, to achieve a fully developed flow through the domain. Afterwards the wave kinematics is sampled at different depths and stored into binary files. For each depth, we stored the velocities and their spatial gradients, the kinematics (Eulerian) acceleration, the surface elevation and its spatial gradients.

The nondimensional water depth and significant wave height were computed, for each sampled depth, as:

$$
\begin{aligned}
h^{*} & =\frac{h}{g T_{P}^{2}} \\
H_{S}^{*} & =\frac{H_{S}}{g T_{P}^{2}}
\end{aligned}
$$

In the above equation, the significant wave height was calculated as four times the standard deviation of the wave elevation time series $H_{S}=4 \cdot \sigma_{\eta}$. The peak period $T_{P}$ was defined as the period at which the power spectral density curve (PSD) achieved its maximum. The PSD curve was conveniently smoothed via a moving average filter (with a width of $W_{M A}=N_{t} / 500$, where $N_{t}$ was the number of time steps in the wave elevation time series) to simplify the identification of the PSD peak.

The bidimensional computational domain was discretized with $N_{X}=2^{14}+1$ cells in the wave direction, and $N_{Z}=17$ points in the depth direction. While the grid was regular in the $\mathrm{x}$-wise direction, the vertical grid spacing was not even, due to the shoaling domain and to the discretization strategy. OceanWave3D then uses a so-called $\sigma$-transformation to solve the Laplace equations on an equivalent regular grid. Further details on how the flow governing equations are solved are available in the paper from Engsig-Karup et al. [11.

The time step for the simulation was $\Delta t=0.07[s]$, which guaranteed a Courant Number lower than 1 in the whole domain.

The incoming spectra were of the JONSWAP type [18. The peak enhancemente factor $\gamma$ was derived according to the DNV recommended practices [19]. To achieve a satisfactory description of all the waves in the spectrum, it was decided to resolve the shortest waves with at least 9 grid points in the $\mathrm{x}$-direction. Hence the spectrum high-cut frequency was set at $T_{h c}=3.0[s]$. The spectrum was then discretized into $2^{19}$ components in the frequency axis, to achieve a $\Delta t=0.07[s]$, which guaranteed a return time of roughly 10 hours.

For each sea state, 10 different realizations were performed. For each of these realizations, the amplitude of the discrete components was always the same, defined by the above mentioned JONSWAP spectrum. On the contrary, their phases were each time different, as they were randomly sampled from a uniform distribution.

A simple breaking model is present in the fully nonlinear code. As a potential code cannot directly model breaking, some energy is subtracted from the domain when the surface vertical downward particle acceleration $d w / d t$ overcomes a certain threshold. Following the indications in [20, in the current work the limit value was set to $-0.5 g$, where $g=9.80665\left[\mathrm{~m} / \mathrm{s}^{2}\right]$. 


\section{THE RAINEY FORCE MODEL}

One the wave kinematics are known, a time series of the $\mathrm{x}$-wise force on a stiff cylinder was computed by means of the Rainey [13, force model. The distributed force on the cylinder can be computed as :

$$
F_{d}[N / m]=\frac{1}{2} \rho C_{D} u|u|+\rho \pi\left(1+C_{m}\right) R^{2} \frac{\mathrm{d} u}{\mathrm{~d} t}
$$

In the above equation, $\rho$ is the fluid density, $C_{D}$ is the drag coefficient, $u$ is the streamwise fluid velocity, $C_{m}$ is the added mass coefficient, $R=3.5[\mathrm{~m}]$ is the cylinder radius, and $\mathrm{d} u / \mathrm{d} t$ is the total (material) derivative of the streamwise velocity.

In addition to the above, a so-called axial divergence distributed force is predicted by the Rainey model:

$$
F_{a d}[N / m]=\rho \pi R^{2} C_{m} \underline{\eta}_{z} u
$$

In this equation, $\underline{\eta}_{z}=\partial w / \partial z$ is the z-wise spatial derivative of the vertical fluid velocity $w$. This force accounts for the fact that, even though the cylinder is slender in the horizontal direction with respect to the wavelength, it is not slender in the vertical direction. To obtain the force at the monopile base, the distributed force contributions were depth-integrated from the bottom of the monopile $(z=-h)$ to the time-varying surface elevation $(z=\eta(t))$.

One last point force needs to be added at the intersection between the surface and the cylinder, to take into account for the change of fluid kinetic energy which happens when the wet length of the cylinder varies:

$$
F_{p}[N]=\frac{1}{2} \rho \pi R^{2} C_{m} \eta_{x} u^{2}
$$

In the above equation, $\eta_{x}=\partial \eta / \partial x$ is the $\mathrm{x}$-wise slope of the surface elevation. This load model is derived on energy conservation grounds only, and therefore it does not make any assumptions on the incoming waves, except the fact that the cylinder is slender and does not modify the wave surface (the so-called wavy lid assumption).

To find values for $C_{m}$ and $C_{D}$ for the smooth steel cylinder, a designer normally reslies on recommended practices, as the ones in DNV-RP-C205 (see 6-7 and 6-9 in [19]). Typical values for a smooth cylinder at sub-critical Reynolds numbers are $C_{m}=1.0$ and $C_{D}=1.0$. In our work, the choice of these two parameters is vital to achieve a good comparison between simulations and experiments, and will be discussed in the Results section.

\section{DISPERSION OF THE SIMULATION RESULTS DUE TO RANDOMNESS}

As above mentioned, for each combination of $H_{S}$ and $T_{P}, 10$ different random realizations were performed. This led to a dispersion of the simulated statistical 


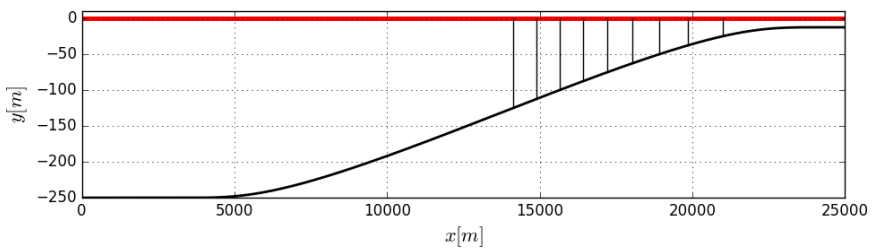

Figure 1: Computational domain with sampling depths locations. Sampling depths are from $125[\mathrm{~m}]$ to $25[\mathrm{~m}]$, with a $25[\mathrm{~m}]$ interval.

quantities at the locations where the kinematics was sampled.

To give an indication of the spread of the statistical parameters in the simulated time series, we present the calculation of the significant wave height $H_{S}$ and the standard deviation of the x-wise force $\sigma_{F_{x}}$ for 10 realizations, when a JONSWAP spectrum with $H_{S}=15.77[\mathrm{~m}], T_{P}=15.15[\mathrm{~s}], \gamma=3.9$ was imposed at the wave generation zone. The kinematics was sampled at $h=25.0[\mathrm{~m}]$, and the excitation force on a cylinder was computed by means of Equations 5,6 and 7 (i.e. without slamming force). The draft of the cylinder used for this example force computations was also $h=25.0[\mathrm{~m}]$ and its diameter was $D=7.0[\mathrm{~m}]$ (note that in the results section we will use a draft of $h=20.0[\mathrm{~m}]$ ). For this investigation we chose a $C_{m}=1$ and $C_{D}=1$. The results are summarized in Table 1. The Simulated Time is the length of the simulation in the physical scale, i.e. how many second of simulation we were able to sample, after the 2-hours transient was disregarded.

We remark that one simulation did not manage to run for longer than the warm-up time, therefore we marked it with $\mathrm{N} / \mathrm{C}$, standing for Not Converged. The two shortest simulations $(\approx 6700[s]$ and $\approx 11400[s])$ also broke down earlier than expected. We believe that some combinations of the random phases give rise to particularly steep waves that the potential solver cannot handle, despite the stabilizing effect of the breaking model. This is also the reason why the simulated time is not the same for the other 7 seeds, despite the employed computational (CPU) time was the same. We believe the solver was employing a marginally larger amount of computational time to reach convergence at every time step when particularly steep waves arose in a certain realization. Overall, this led to a shorter simulated time for the same CPU time.

The dispersion around the mean of the 10 sampled $H_{S}$ was $\sigma_{H_{S}}=0.292[\mathrm{~m}]$. In fact, the significant wave height is sensitive to the number of breaking waves in a particular realization. For every realization, since the number of breaking events depends on the random superimposition of the single waves components, the sampled significant wave height has a certain dispersion. As for the $\mathrm{x}$-wise force, a similar trend is observed, and the standard deviation in the force signal is positively correlated with the sampled $H_{S}$. The ratio between the standard deviation and the mean, for both $H_{S}$ ahd $F_{x}$, was close to 0.02 , indicating that the spread in the energy of the wave elevation and force signals was, in fact, relatively small. 


\begin{tabular}{cccc}
\hline Seed N. & $H_{S}[m]$ & $\sigma_{F_{x}}[M N]$ & Simulated Time $[s]$ \\
\hline 1 & 14.10 & 2.71 & 25094 \\
2 & 13.71 & 2.64 & 25217 \\
3 & 13.73 & 2.65 & 28008 \\
4 & 14.27 & 2.76 & 25809 \\
5 & 14.17 & 2.73 & 27693 \\
6 & 14.43 & 2.79 & 11415 \\
7 & 14.19 & 2.74 & 25058 \\
8 & 14.25 & 2.75 & 25401 \\
9 & 14.69 & 2.85 & 6744 \\
10 & $\mathrm{~N} / \mathrm{C}$ & $\mathrm{N} / \mathrm{C}$ & $\mathrm{N} / \mathrm{C}$ \\
\hline$\square$ & 14.17 & 2.74 & - \\
$\sigma_{\square}$ & 0.292 & 0.0614 & - \\
$\sigma_{\square} / \bar{\square}$ & 0.0206 & 0.0224 & - \\
\hline
\end{tabular}

Table 1: Example of significant wave height $H_{S}$ and of the standard deviation of the relative x-wise excitation force $\sigma F_{x}$, calculated for a monopile with a diameter of $D=7.0[\mathrm{~m}]$ and positioned at a water depth of $h=25.0[\mathrm{~m}]$. The values are relative to 10 different realizations, obtained by using the same deep water JONSWAP spectrum with $H_{S}=15.77[\mathrm{~m}], T_{P}=$ $15.15[s]$ and a peakedness factor of $\gamma=3.9$, but where the random phases of the wavelets were chosen by sampling a uniform distribution. One of the simulations did not converge $(\mathrm{N} / \mathrm{C})$. The total simulated time for each seed (excluding the 2 hours warm-up period) is reported in the last column.

\section{THE PRESSURE IMPULSE MODEL}

The slamming force from waves is often modelled with the Wienke and Oumeraci model [8]. The accuracy of this model has often been assessed in terms of maximum force, i.e. the maximum value of the overall wave force plus the slamming contribution from the impact load model. For structural modes with high frequency relative to the fundamental wave frequency, the structural response is governed by the force impulse (i.e. the time integral of the force), rather than the peak force value. Hence, even with a reliable prediction of the peak force, the response will still depend on an accurate description of the impact time scale.

A simpler approach to the slamming loads may thus be obtained by direct modelling of the time integrated force. The recent pressure impulse model of Ghadirian and Bredmose 15 implements this approach by using a closed-form solution to a boundary value problem defined by simple wave parameters. The solution (equation 2.9 in 15 ) is the pressure impulse field (time integrated impact pressure) on the cylinder face, which can further be integrated over the structure to achieve the force impulse. The spatial distribution of the pressure impulse is thus part of the solution.

We describe the model briefly here. The fluid domain and its boundary conditions are shown in Figure 2, The impacting wave is described in an idealized geometry. The domain is wedge-shaped with with azimuth limits $-\theta_{\max } \leq \theta \leq \theta_{\max }$ and consists of initially moving and still fluid above and 
below $z=-\mu H$ respectively. Here $H$ is the vertical distance from the bed to the free surface for the wave at the initial impact and $\mu H$ is the vertical height of the impact zone. Until before the time of impact, the upper part is approaching the cylinder with velocity $U$ in the negative $x(r \cos \theta)$ direction. At the boundaries $\theta= \pm \theta_{\max }, z=0$ and $r=b$ the condition $P=0$ has to be satisfied. The Laplace equation is solved in the cylindrical coordinate system to yield

$$
\begin{aligned}
P=\sum_{m=1}^{\infty} \sum_{n=1}^{\infty}\left(A_{m n} \cos \left(L_{m} \theta / \theta_{\text {max }}\right) \sin \left(k_{n} \frac{z}{H}\right)\right. & \left.\frac{I_{q_{m}}\left(k_{n} \frac{r}{H}\right)+\alpha_{m n} K_{q_{m}}\left(k_{n} \frac{r}{H}\right)}{\partial_{r}\left(I_{q_{m}}\left(k_{n} \frac{r}{H}\right)+\alpha_{m n} K_{q_{m}}\left(k_{n} \frac{r}{H}\right)\right)_{r=a}}\right),
\end{aligned}
$$

where $q_{m}=L_{m} / \theta_{\max }$ is the order of the Bessel functions, $k_{n}=(n-1 / 2) \pi$ and $L_{m}=(m-1 / 2) \pi$. Further $\alpha_{m n}$ is chosen such that $P=0$ at $r=b$ and

$$
A_{m n}=\frac{2 \rho U}{\theta_{\max }} \frac{1-\cos \left(k_{n} \mu\right)}{k_{n}} \int_{-\theta_{\max }}^{\theta_{\max }} \cos (\theta) \cos \left(L_{m} \theta / \theta_{\max }\right) d \theta d z .
$$

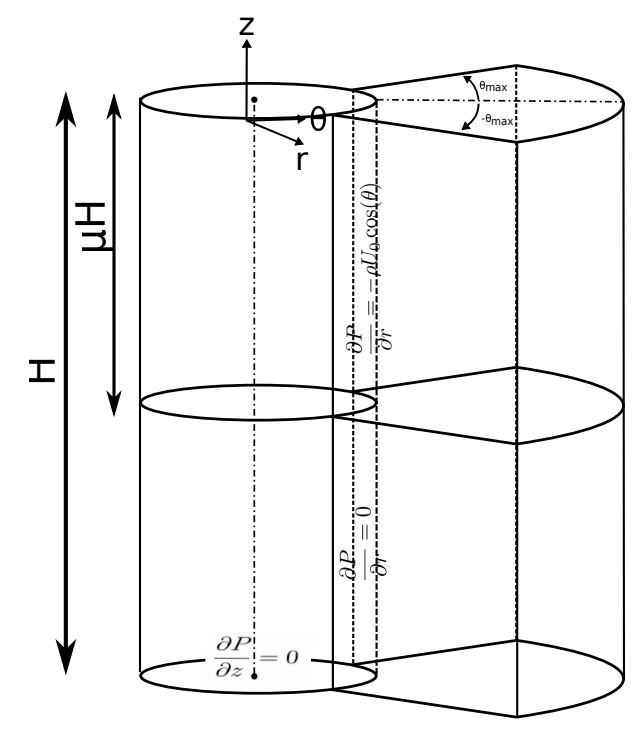

Figure 2: Definition sketch for wedge-shaped 3D impact on a vertical cylinder.

From this equation, the non-dimensional pressure impulse is seen to depend on the normalized outer radius, $b / H$, impact height $\mu$, cylinder radius $a / b$ and the maximum impact angle $\theta_{\max }$ :

$$
\frac{P}{\rho U H}\left(\frac{r}{H}, \theta, \frac{z}{H}\right)=f\left(\frac{b}{H}, \mu, \frac{a}{b}, \theta_{\max }\right) .
$$




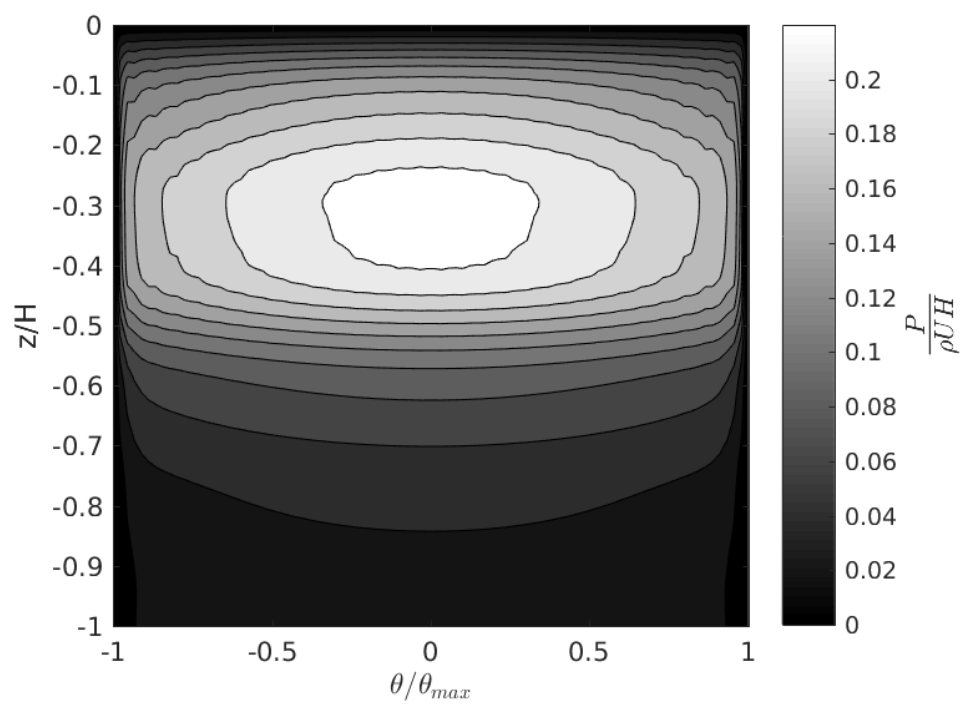

Figure 3: Example of the pressure impulse distribution on the cylinder wall.

In Figure 3 an example distribution of the pressure impulse on the cylinder is shown for $b / H=5, \mu=0.5, a / b=0.5$ and $\theta_{\max }=\pi / 4$. The effect of each parameter was investigated in [15]. It was concluded that $\theta_{\max }$ was the only parameter not determinable from the incident wave. A value of $\pi / 4$ was found to provide a good match to the CFD impact analysed. Initial results for validation against model tests was presented by Ghadiran and Bredmose [16. Here, the force impulse was determined for 7 slamming events from the DHI DeRisk experiments (see next section) and reproduced by the pressure impulse model. A good comparison was achieved with $\theta_{\max }=\pi / 5$. The difference with the above mentioned optimal value of $\pi / 4$ may be a consequence of the underestimation of the experimental force impulse by the chosen detection method. An aggressive Butterworth low-pass filter was used to estimate the inline force time series excluding the effect of the slamming. The same method of estimation of the force impulse in the measurements is used in the present work, hence $\theta_{\max }=\pi / 5$ is chosen.

\section{MODEL TESTS}

The model tests that were used for comparison in the current work were performed at DHI in 2015 in the framework of the DeRisk project. The experiments aimed at reproducing storms with different return times (10 to 1000 years) for two typical northern sea locations $(20[\mathrm{~m}]$ and $33[\mathrm{~m}]$ depth). Values in this section are hereby reported at full scale, which is 50:1 with respect to model (or laboratory) scale. For a conversion of some parameters to model scale, see 


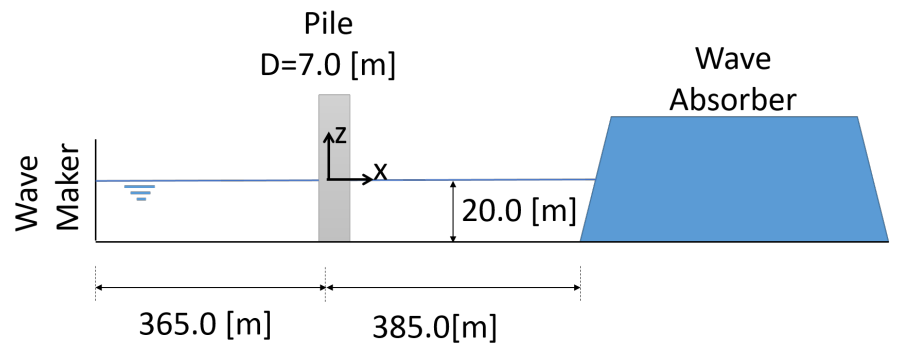

Figure 4: Experimental setup. Elaboration of Figure 1 in 20. The dimensions in this picture are reported in full scale, which is 50:1 with respect to model (laboratory) scale.

Table 2. In Figure 4, we sketched the experimental setup for the experiments analyzed in the current work. The wave tank was $W=1250[\mathrm{~m}]$ wide and $L=1650[\mathrm{~m}]$ long, with a flat bottom. The effective length of the basin was reduced by positioning crushed stones behind the cylinder, to mitigate wave reflection. The basin's wave maker was made up of 36 wave paddles, hinged at the bottom, and was capable of generating both $2 \mathrm{D}$ and $3 \mathrm{D}$ waves. A steel cylinder with a diameter of $D=7.0[\mathrm{~m}]$ was positioned $365.0[\mathrm{~m}]$ away from the paddles.

An array of $5 \times 5$ wave gauges was positioned around the cylinder with a spatial resolution of $10[\mathrm{~m}]$. Five additional wave gauges were positioned in the space between the wave gauge array and the wave paddle generator, see Figure 5.

The cylinder was connected to two force transducers, one at the top and one at the bottom of the cylinder, and allowed for the measurement of the loads in all six degrees of freedom, sampled every $0.01[s]$. The first bending eigenfrequency of the transducers and cylinder assembly was tuned to be far away from the wave spectrum frequencies, in order to avoid pollution of the wave force signal by the structural response.

\begin{tabular}{ccc}
\hline Parameter & Model scale & Full scale \\
\hline$R[m]$ & 0.14 & 7 \\
Depth $[m]$ & 0.4 & 20 \\
Basin Width $[m]$ & 25 & 1250 \\
Basil Length $[m]$ & 33 & 1650 \\
\hline
\end{tabular}

Table 2: Conversion from model (laboratory) scale to full scale for some parameters. The experiments were designed to have a 1:50 scale with respect to full scale.

\section{THE CHOSEN TIME SERIES}

One particular experiment was chosen for the validation of the simulation results. This sea state, relative to the location with $20[\mathrm{~m}]$ water depth, had a 


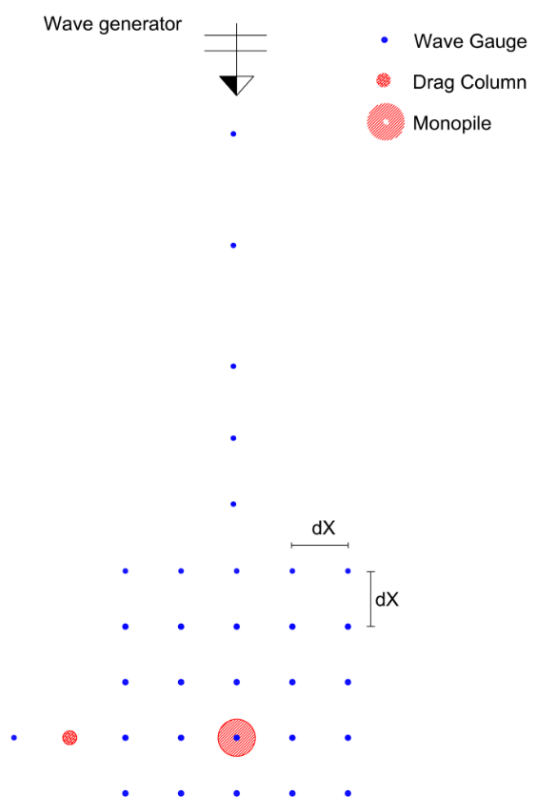

Figure 5: Position of the wave gages and of the cylinder in the wave tank. The distance between the $5 \times 5$ array of wave gages is $\Delta x=10.0[\mathrm{~m}]$. The distance between the cylinder and the wave generator is $365.0[\mathrm{~m}]$. 


\begin{tabular}{ccccccc}
\hline & $H_{S}[m]$ & $T_{P}[s]$ & $\gamma$ & $\mathrm{h}[m]$ & $H_{S}^{*} \cdot 10^{3}$ & $h^{*} \cdot 10^{2}$ \\
\hline $\operatorname{Exp}$ & 7.65 & 14.06 & 1. & 20 & 3.95 & 1.03 \\
\hline$S_{1}$ & 7.58 & 14.25 & 1.75 & 20 & 3.81 & 1.00 \\
$S_{2}$ & 7.71 & 14.17 & 1.75 & 20 & 3.92 & 1.02 \\
$S_{3}$ & 7.76 & 14.19 & 1.75 & 20 & 3.93 & 1.01 \\
$S_{4}$ & 7.33 & 13.86 & 1.75 & 20 & 3.89 & 1.06 \\
$S_{5}$ & 7.31 & 14.06 & 1.75 & 20 & 3.77 & 1.03 \\
\hline
\end{tabular}

Table 3: Scaled simulations. The parameter $\gamma$ is the peak enhancement factor that was used to generate the JONSWAP spectra. We can see that, after the scaling, the simulations $H_{S}$ and $T_{P}$ show a certain scatter around the experimental values.

1000 year return time. The parameters in full scale are summarized in the first row of Table 3: $H_{S}=7.65[\mathrm{~m}]$ and $T_{P}=14.06[\mathrm{~s}]$. The measured time series is 6 hours long.

This experimental time series was chosen since it had the largest number of breaking event. Also, we could identify a good number of similar simulations in the $\left(H_{S}^{*}, h^{*}\right)$ plane, which allowed for a meaningful comparison. Among the Froude-similar simulations, we selected the 5 that were closest to the experiment. For other experiments, where only a few Froude-similar simulations were found among the computed time series, the comparison was less good.

In order to Froude-scale the simulations to match the scale at which the experiments were performed, we calculated a scale factor $\lambda$ by taking the ratio of the experimental and simulated depth:

$$
\lambda=\left(\frac{h^{e x p}}{h^{s i m}}\right)
$$

Once the scaling factor is calculated, the simulated velocity, wave elevation, acceleration time series are Froude-scaled. Also the time scale is corrected according to the Froude law. The total simulated time among all the 5 selected computations was 30 hours. The scaled results are summarized in Table 3.

In practice, a different scaling would be possible. One could calculate the $\lambda$ factor by taking the square of the ratio between the peak periods, or by taking the ratio of the significant wave heights. However, we decided to use the ratio between the depth as this was more straight-forward to define and as it led to a consistent force integration, since it fixed the water deptht to $20[\mathrm{~m}]$.

We remark that even though the peak shape parameter of the input JONSWAP spectra was chosen according to best practices [19] both for the simulations and for the experiments, the evolution of the wave spectra across the experimental and the computational domain led to a different redistribution of the energy. The experiments were in fact performed on a flat bed, and the waves were generated via paddle motion, while the simulations were run on a sloping bed and the waves were imposed in a relaxation zone. This led to remarkably different spectra at the location of the comparison, which explains the difference 
between the peak shape parameter $\gamma$ in the experimental and in the simulated storms, see Table 3

In our methodology, we did not perform simulations in order to reproduce a particular experimental test case, but we rather chose the simulations which were relevant for the comparison ex-post, according to their nondimensional significant wave height and water depth $\left(H_{S}^{*}, h^{*}\right)$. The difference in peak shape parameter shows that, even though the sea states were nondimensionally similar and despite having a similar standard deviation, more energy was concentrated around the peak period in the simulated spectra $(\gamma=1.75$, meaning a sharper spectral peak $)$ than in the experimental one $(\gamma=1$, meaning a wider spectral peak).

The difference between the energy distribution in the experimental and the simulated spectra will be evident in the Results section of this paper.

\section{RESULTS}

In Figure 6(a), we can observe an excerpt of the simulated and experimental time series plotted on top of each other. In all the following comparisons, the red color denotes experiments, and the blue color denotes each of the 5 simulations chosen for comparison. In order to obtain a total representative simulated signal, we decided to stack the single simulations one after the other, to finally obtain the green colored time series.

In Figure 6(c), the spectral peak periods of the simulated and of the overall computed simulations show a very good match. However, the standard deviation of the experimental time series is $2 \%$ higher than in the computational one. Although we have tried to select simulations that were, in nondimensional terms, similar to the experiment, the nondimensional significant wave height and nondimensional depth of the simulation were slightly dispersed around the experimental value, as from table Table 3. Therefore, when deciding to scale according to Equation 11, we implicitly accepted a compromise on the $H_{S}$ and $T_{P}$ scaling, which led to the observed discrepancy.

The shape of the spectra disagree quite strongly for frequencies larger than $0.1[H z]$, where the experiment exhibits clear peaks at two superharmonics. These secondary peaks are located at frequencies that are not multiple of the spectral peak frequency $f_{P}=1 / T_{P}=0.071[H z]$. In a paper by Schløer et al. 20 the experiments were reproduced by OceanWave3D in a domain that was physically equivalent to the wave tank and where a numerical wave paddle used the experimental wave paddle signal to produce waves in the numerical tank. Under these conditions, they observed peaks in the wave elevation spectrum at the same locations. This indicates that these peaks are likely linked to local effects due to the domain configuration and to the wave generation strategy.

In Figure 6(b), the experimental wave elevation peak exceedance probability agrees very well with the simulated results down to an exceedance probability level $(P)$ of $10^{-2}$. For less likely events, they start to deviate, and the experimental curve lies above the simulated overall signal. A first explanation can be 

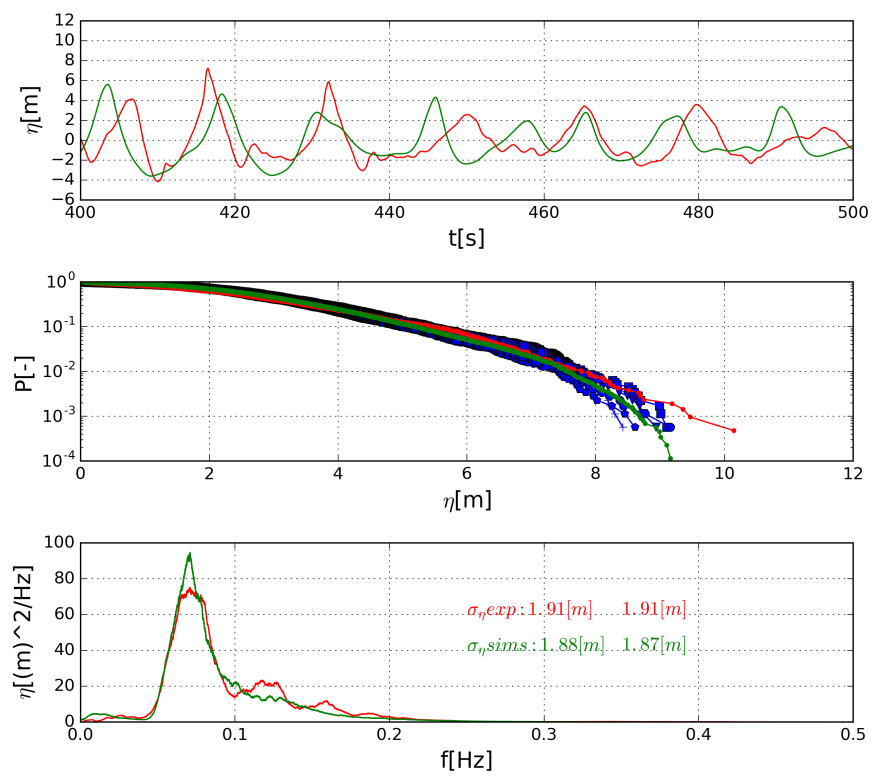

Figure 6: Comparison of the experimental and numerical wave elevation parameters. Experiments are in red, simulations in green. In Figure (a), time excerpts of the wave elevation time series are plotted. In Figure (b), the peak exceedance probabilities, we also report the single exceedance probability curves relative to each of the 5 simulations that were stacked together to we get the green total signal. The two columns of text values in Figure (c) represent the standard deviation of the experimental and the computational values. The values on the left are computed by the square root of the area below the spectra, while the ones on the right were computed by taking the standard deviation of the time domain signal.

found in the different energy found in the the experimental and numerical signal. Secondly, part of the deviations might be caused by the numerical breaking filter which overestimates the onset of breaking with respect to the experiments.

For the current sea state, this would have led to a $C_{m}=1.0$ and $C_{D}=1.0$. For our comparison, this choice would have resulted in a $15 \%$ overestimation of the $\mathrm{x}$-wise simulated force standard deviation with respect to the experimental values. Therefore, we decided to correct the $C_{m}$ so that the standard deviation of the experimental force would match the experimental one, ending up with a $C_{m}=0.79$ and $C_{D}=1.0$. In practice, an engineer designing a wind farm would not have this possibility, since there normally are no experimental results to compare to. However, we decided to follow this approach to make the current comparison more meaningful, and to address the possible causes of the discrepancy in a future study. In particular, we will investigate how the breaking criterion influences the statistics of the extreme waves, and how the different domain shapes (flat versus sloped) and wave generation strategies affect the kinematics at the cylinder location.

Once the force is computed, it is possible to compare it with the measured 

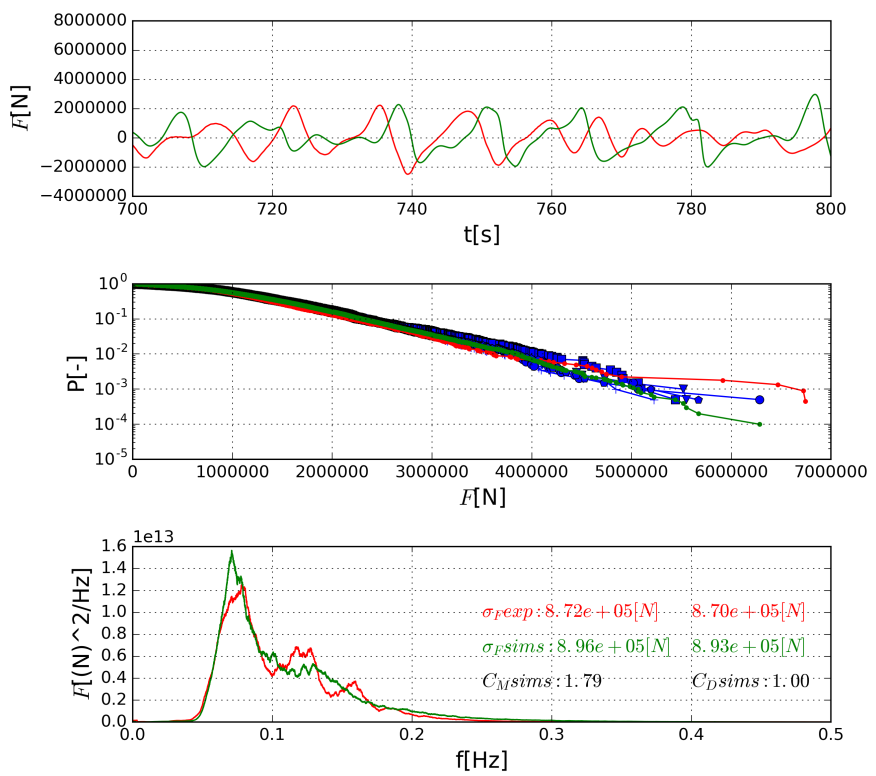

Figure 7: Comparison of the experimental and numerical force signal statistical parameters. Experiments are in red, simulations in green. The two columns of text values in the (c) plot represent the standard deviation of the experimental and the computational values. The values on the left are computed by the square root integrating the area below the spectra , while the ones on the right were computed by taking the standard deviation of the time varying signal.

force, see Figure 7 After the $C_{m}$ correction, the simulations predict a $2 \%$ higher standard deviation in the simulated force signal than in the experimental one. The spectral energy is distributed similarly to what noted for the surface elevation, which, as mentioned, can be traced back to the domain configuration and wave generation strategy. This said, we notice a quite good agreement between the computed force peak exceedance probability and the experimental one for the main population, down to a probability level of $10^{-2}$. For the largest events, the $\mathrm{x}$-wise simulated force is significantly lower than the experimental one. From the analysis of the experimental force time series, we could observe that the events in the tail of the experimental distribution are in fact likely to be slamming events. Since the hitherto computed force signals do not include any slamming calculation, we indeed expected an underestimation of the magnitude of these extreme events.

In the following section, we will use the pressure impulse model to add the slamming forces and try to achieve a more complete picture of the load spectrum on the cylinder. 


\section{APPLICATION OF THE PRESSURE IMPULSE MODEL}

We now pursue a method to add a slamming contribution on top of the kinematicsbased force. In a general design case, no experimental results will be available. The method must thus be based on the incident wave kinematics alone. We here present our first attempt for such a method and its calibration against model tests.

First, experimental values of the force impulse was extracted from the chosen test series, which was 6 hours long. From the inline force time series the 30 waves with the largest peak values were selected and then visually investigated to select the waves which generated slamming loads. With this method 7 slamming events from the measurements were detected. Next, the experimental force impulse was calculated by the same method as used by Ghadirian and Bredmose [16]. In this approach, the slamming part of the inline force time series are isolated by using the Butterworth filtering extensively. The area between the filtered force time series and the non-filtered one is taken as the force impulse from the experiments.

Next, a method to detect the waves in the numerical domain that would slam on the structure was developed. The condition for choosing these events was that the breaking filter in OceanWave3D is active closest to and upstream of the cylinder. This is a standard output of the OceanWave3D model. As mentioned, the breaking filter is active when the downward vertical particle acceleration exceeds a threshold $\mathrm{d} w / \mathrm{d} t<-0.5 \mathrm{~g}$. The force impulse for all the detected events was next calculated as follows. The fluid thickness, $H$, was calculated as the depth plus the maximum crest height of the wave and $a$ is the radius of the cylinder $(D=7.0[m])$. For the outer radius $b$, it was shown in Ghadirian and Bredmose 2019 [15] that the results of the pressure impulse model are not sensitive when it is larger than an asymptotic value. This was also the case in the present study, so $b$ was chosen to be $L_{p} / 2$ where $L_{p}$ is the peak period wave length using the linear dispersion relation.

Further, as a simple choice, the impact zone height was taken equal to the distance between the still water level and the maximum crest height for the breaking event

$$
\mu H=\eta_{\max }
$$

The event which included $\mu$ smaller than 0.1 were excluded since the low values of the $\mu$ shows that the numerical breaking filter has dissipated the wave completely and we did not have access to get the full description for such waves. For consistency with the experimental force impulse detection and based on the results of Ghadirian and Bredmose 2019 [16], the parameter $\theta_{\max }$ was chosen to be equal to $\pi / 5$.

The last model parameter, the impact velocity $U$, was chosen to be $-\eta_{t} / \eta_{x}$, at $0.1 \cdot T_{p}[s]$ before the instant when the integrated inline force from Equation 5 was maximum at the monopile. This method was used to estimate the velocity of the crest of the waves. 


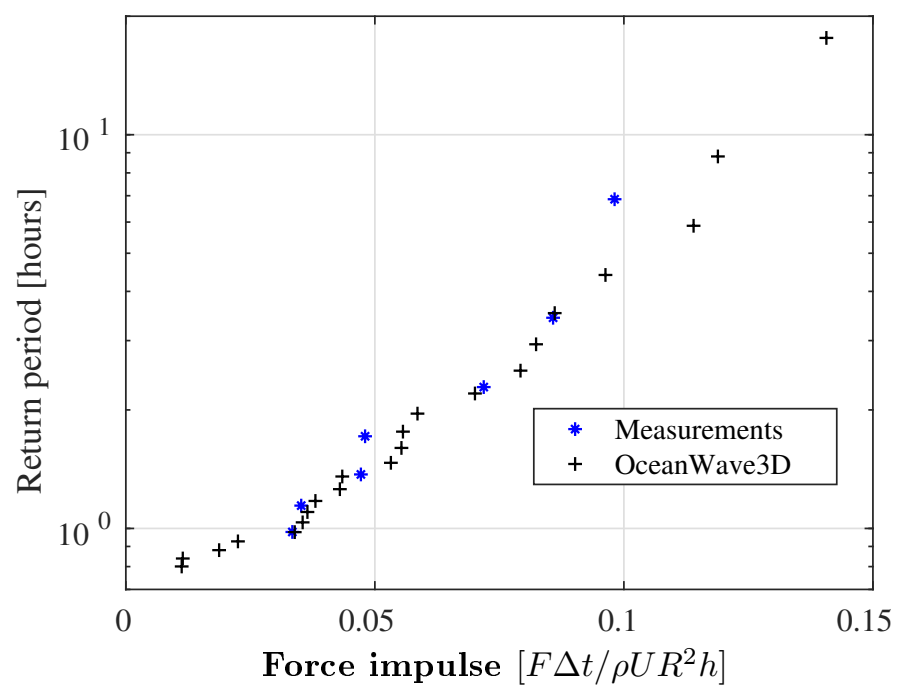

Figure 8: Comparison of experimental (measurements) and numerical force impulse in terms of 1-hour exceedance probabilities. The force impulses were nondimensionalized with respect to $\rho U h R^{2}$.

Based on the ratio of the experimental time series ( 6 hours) and the numerical time series (23 hours), 26 events were chosen from the numerical results. These 26 events were selected as the events giving the largest force impulses. The experimental and numerical force impulses are compared in Figure 8 in terms of the 1 hour exceedance probabilities for the specific sea state, and a good match is observed.

Once the force impulse is calculated following the above methodology, it is possible to compute a force time series that generates that impulse on the structure. One could impose a certain variation profile (e.g. a triangular wave, with an ascending and a descending ramp of the same duration) and a maximum force (e.g. the maximum force from one of the classical models like Goda et al. [7), and then derive the duration of the impulse $\Delta t$. This force time series can be superimposed to the excitation force computed via the Rainey model, to finally obtain a physically sound and comprehensive force model. The details of this procedure are outside of the scope of the current paper, and will be subject of future investigations.

\section{CONCLUSIONS}

In this paper, we have presented an initial comparison of forces by precomputation of kinematics and experiments. We have also demonstrated Froude scaling and its potential for extending the validity of the precomputed kinematics.

When comparing experiments and the 5 selected simulations from the precomputed set, the surface elevation spectra matched reasonably well. The en- 
ergy content of the two signals was however quite different, which can be ascribed to the difference in the domain shape and wave generation strategies. As for the experimental and simulated $\mathrm{x}$-wise force, the main population of the exceedance probability distribution matched well. On the other hand, the simulated events in the tail of the distribution had a smaller magnitude than the corresponding experimental ones.

To overcome this limitation, we further presented a method for adding slamming loads from a new pressure impulse model to the simulation results. The procedure is based on incident wave kinematics only, and can thus be applied together with the information available from the precomputed kinematics. The model parameters were extracted from the incident waves, and by the proposed choice of the impact velocity a good match between the experimental and numerical force impulses was found.

The above results can be improved for example by a more accurate detection of the slamming events in the experimental data. They however show the potential of the combined application of precomputed incident wave kinematics and the pressure impulse model. Further validation is part of our present work, and will be reported as further output of the DeRisk project.

\section{ACKNOWLEDGEMENT}

This work was funded by the Innovation Fund Denmark and other partners as part of DeRisk project with grant number 4106-00038B. This support is gratefully acknowledged by the authors.

\section{References}

[1] Oggiano, L., Pierella, F., Vaal, J. D., Nygaard, T. A., Stenbro, R., and Arens, E., 2017. "Modeling of 2D Irregular Waves on a Sloped Bottom Using a Fully Nonlinear Navier-Stokes / VOF F ormulation". In Proceedings of the International Offshore and Polar Engineering Conference, pp. 622629.

[2] Bredmose, H., Mariegaard, J., Paulsen, B. T., Jensen, B., Schløer, S., Larsen, T., Kim, T., and Hansen, A. M., 2013. The Wave Loads project. Tech. rep., DTU Wind Energy.

[3] International Electrotechnical Commission. Wind energy generation systems - part 3-1: Design requirements for fixed offshore wind turbines. Tech. rep., IEC, Geneva, CH.

[4] Fenton, J., 1990. "Nonlinear wave theories". The Sea-Ocean Engineering Science, $\boldsymbol{9}(1)$, pp. 3-25.

[5] Pierella, F., Stenbro, R., Oggiano, L., Vaal, J. D., and Nygaard, T. A., 2017. "Stream Function Wave Embedment into Linear Irregular Seas : 
A New Method Based on the Hilbert Transform". In Proceedings of the International Offshore and Polar Engineering Conference, pp. 412-419.

[6] Rainey, P. J., and Camp, T. R., 2007. "Constrained non-linear waves for offshore wind turbine design". In Journal of Physics, Vol. 75, IOP Publishing, p. 12067.

[7] Goda, Y., Haranaka, S., and Kitahata, M., 1966. "Study on impulsive breaking wave forces on piles". Report Port and Harbour Technical Research Institute, 6(5), pp. 1-30.

[8] Wienke, J., and Oumeraci, H., 2005. "Breaking wave impact force on a vertical and inclined slender pile - Theoretical and large-scale model investigations". Coastal Engineering, 52(5), pp. 435-462.

[9] Hallowell, S., Myers, A. T., and Arwade, S. R., 2016. "Variability of breaking wave characteristics and impact loads on offshore wind turbines supported by monopiles". Wind Energy, $\mathbf{1 9 ( 2 ) , ~ p p . ~ 3 0 1 - 3 1 2 . ~}$

[10] de Ridder, E. J., Bunnik, T., Peeringa, J. M., Paulsen, B. T., Wehmeyer, C., Gujer, P., and Asp, E., 2017. "Summary of the Joint Industry Project Wave Impact on Fixed Foundations (WiFi JIP)". In Volume 10: Ocean Renewable Energy, ASME, p. V010T09A081.

[11] Engsig-Karup, A. P., Bingham, H. B., and Lindberg, O., 2009. "An efficient flexible-order model for 3D nonlinear water waves". Journal of Computational Physics, 228(6), pp. 2100-2118.

[12] Morison, J., Johnson, J., and Schaaf, S., 1950. "The Force Exerted by Surface Waves on Piles". Journal of Petroleum Technology, 2(05), pp. 149154.

[13] Rainey, R. C. T., 1995. "Slender-Body Expressions for the Wave Load on Offshore Structures". Proceedings of the Royal Society A: Mathematical, Physical and Engineering Sciences, 450(1939), pp. 391-416.

[14] Kok Yan Chan, R., 2016. "Computation of Nonlinear Hydrodynamic Loads on Floating Wind Turbines using Fluid-Impulse Theory". Phd thesis, Massachussets Institute of Technology.

[15] Ghadirian, A., and Bredmose, H., 2019. "Pressure impulse theory for a slamming wave on a vertical circular cylinder". Journal of Fluid Mechanics, 867, pp. R1-1 - R1-14.

[16] Ghadirian, A., and Bredmose, H., 2019. "Initial experimental validation of a pressure impulse model for a vertical circular cylinder". In Proceedings of the International Workshop on Water Waves and Floating Bodies 2019, Newcastle University, ed. 
[17] Bredmose, H., Dixen, M., Ghadirian, A., Larsen, T. J., Schløer, S., Andersen, S. J., Wang, S., Bingham, H. B., Lindberg, O., Christensen, E. D., Vested, M. H., Carstensen, S., Engsig-Karup, A. P., Petersen, O. S., Hansen, H. F., Mariegaard, J. S., Taylor, P. H., Adcock, T. A., Obhrai, C., Gudmestad, O. T., Tarp-Johansen, N. J., Meyer, C. P., Krokstad, J. R., Suja-Thauvin, L., Hanson, T. D., Torben, J., Harry, B., Damgaard, E., Peter, A., Skov, O., Sandvig, J., and Jacob, N., 2016. "DeRisk - Accurate Prediction of ULS Wave Loads. Outlook and First Results". In Energy Procedia, Vol. 94, Elsevier, pp. 379-387.

[18] Hasselmann, K., Barnett, T., Bouws, E., Carlson, H., Cartwright, D., Enke, K., Ewing, J., Gienapp, H., Hasselmann, D., Kruseman, P., Meerburg, A., Müller, P., Olbers, D., Richter, K., Sell, W., and Walden, H., 1973. "Measurements of wind-wave growth and swell decay during the Joint North Sea Wave Project (JONSWAP)". Ergänzungsheft, $8(12)$.

[19] Det Norske Veritas, 2010. Environmental conditions and environmental loads. Tech. Rep. October, DNV.

[20] Schløer, S., Bredmose, H., and Ghadirian, A., 2017. "Experimental and Numerical Statistics of Storm Wave Forces on a Monopile in Uni- and Multidirectional Seas". In Volume 10: Ocean Renewable Energy, ASME, p. V010T09A073. 